\section{Dr. Berdine,}

Thank you for putting together an interesting and informative presentation on such a weighty topic. I imagine that this could easily be a series of presentations where each "chapter" of your original presentation could be expanded upon. More "chapters" could be added as well. I'm personally fascinated by the idea of human augmentation and I'm curious about the idea that future doctors will be more technician than clinician. I wonder if this is happening to a degree already as pacemakers, defibrillators and 3D printed devices become more and more complex (akin to your "what is me?" slide). Anyway, I have two thoughts/questions l'd like you to consider. They can be thought of as responses to your presentation/slide that is shown at time $34: 12$.

1. I believe the genesis of the popularity of quantum consciousness comes from the following statement describing the common human response to something unknown: "I understand neither quantum mechanics nor consciousness - therefore they must be connected." However, just because we don't understand either one this doesn't mean that they are connected in any way whatsoever despite the massive effort to do so by highly regarded scientists using well-known mathematical models (Roger Penrose and Godel's Incompleteness Theorem). What seems to be true is that over multiple millennia worth of time, the prime mover has been pushed further outside the circle of determinism again and again, as scientific discovery explains what once before was assigned to the whims of a mystical power. This process is applicable to practically every area of human knowledge. This is not an argument against a prime mover (in favor of determinism) or against mysticism being the explanation of such a prime mover. Rather, history seems to be saying that perhaps we just need more time in order to understand the full truth of both quantum physics and consciousness.

2. Definition of free will: if quantum mechanics is an essential component of consciousness does it really equal "free will" as we commonly define it? If we assume that "free will exists and is explainable by quantum mechanical effects on membrane molecules" then have we placed quantum mechanics in the position of the prime mover? The unpredictable nature of quantum mechanics may "fit" in this role but if the finite result of a quantum effect causes a predictable future change then this should not completely rule out determinism when the common definition of free will is being invoked. In other words, even if quantum mechanics are proven in the future to affect consciousness I don't consider than equal to definitively concluding free will exists.

Please let me know if anything isn't clear. I look forward to hearing your thoughts. Thanks again

\section{Zachary Mulkey, MD, Internal Medicine} $10 / 4 / 2015$

\section{Response to MULKEY}

The two questions are somewhat intertwined, but I will try to answer them separately.

1. The first question asks whether or not the results of quantum events will remain random. Or will we be able to predict the outcome of individual quantum events with certainty. If we can predict quantum events with certainty, and nerve impulses originate from quantum event changes in membrane molecules, then predicting what people do will become like predicting the weather: we understand how it works but the number of variables is so large and small deviations from given inputs lead to very large deviations from future results due to butterfly effects and chaos theory. If Free Will becomes analogous to the weather, then Dr. Cashmore is correct and Free Will is an illusion. One cannot prove that quantum events are truly random - that is without any deterministic explanation - but I am betting that scientific progress will come up against a hard limit here, much like the question of what happened before the Big Bang. The interference 
pattern is real and it does collapse into the particle pattern when one tries to peek at which slit is chosen. An ability to predict the outcome of quantum events would require us to know which slit the electron will pass through before it enters the experiment without disturbing the interference pattern. In any event, as long as politicians do not declare Free Will to be null and void based on Dr. Cashmore's arguments until we are capable of predicting individual quantum events, I will feel better.

Dr. Penrose remains convinced that Godel's Incompleteness Theorem proves that the human mind contains an unprovable axiom. Others disagree, though Godel seems to agree with Penrose. The question, to me, is a bit different, though it may degenerate into Dr. Penrose's argument. The question as I see it is whether a perfect snapshot of the human brain would allow one to predict what a person will do in response to all possible inputs. If I take a snapshot of a computer, I know what it will do given any set of external inputs. I do not think a snapshot of the human brain will tell us any more about what that person will do any more than taking a snapshot of a voting booth will allow you to predict who will win the election. If we succeed in explaining the seeming randomness of quantum events, then quantum events would no longer be a candidate for the explanation of free will, but I will cross that bridge when humanity gets there.

2. The preceding paragraph was related to both questions. You are objecting to explaining free will by a random process. By random, we exclude any deterministic explanation, but random does not seem to fit with our notion of choice and volition. Random quantum events are candidates for the mechanism of free will by virtue of having no deterministic explanation, but that is not enough. We have to believe that Free Will is something outside our physical universe that can push a random event to one choice or another. I am not sure such a thing can be proven or disproven due to the problem of Type I and Type II statistical errors. An experiment would have to be constructed where we reduce an observable event, such as a muscle twitch, to whether or not a membrane molecule changes quantum state. Such a quantum event would be expected to be random like radioactive decay, but our experiment would have to demonstrate that the subject could make the seemingly random process turn up heads 1000 times in a row. A skeptic could still argue that we got lucky. The experimental complications are enormous, because all possible external events that could produce a reflexive twitch by deterministic mechanisms would have to be excluded from the experiment. Given that I suspect over 99\% of human action is explainable by deterministic mechanisms, I am not sure that the experiment is possible to perform.

As I said in response to \#1, I do not seek to prove that Free Will exists. I merely seek to demonstrate that Dr. Cashmore has not proven that Free Will does not exist. My goal is for others to decline to surrender their freedom of action to politicians based on Dr. Cashmore's Lucretian Swerve.

\section{Gilbert Berdine}

10/5/2015 